\title{
Development of nuclear emulsion for muography
}

\author{
Kunihiro Morishima ${ }^{1,2,3,}{ }^{\star}$, Akira Nishio ${ }^{4}$, Masaki Moto ${ }^{4}$, \\ Toshiyuki Nakano ${ }^{3,4}$, Mitsuhiro Nakamura ${ }^{2,3}$ \\ ${ }^{1}$ Institute for Advanced Research (IAR), Nagoya University, Nagoya, Japan \\ ${ }^{2}$ Institute of Materials and Systems for Sustainability (IMaSS), Nagoya University, Nagoya, Japan \\ ${ }^{3}$ Kobayashi-Masukawa Institute for the Origin of Particle and the Universe (KMI), Nagoya University, Nagoya, Japan \\ ${ }^{4}$ Nagoya University, Department of Physics, Nagoya, Japan
}

\section{Article history}

Received July 5, 2016; accepted October 5, 2016.

Subject classification:

Solid Earth: Exploration geophysics, Instruments and techniques.

\begin{abstract}
Muography (cosmic-ray muon radiography) is the non-destructive inspection by using muons produced by interactions between primary cosmic-rays and the Earth's atmosphere. The principle of this technology is the measurement of absorption of incoming cosmic-ray muons, just like $X$-ray imaging technology. A nuclear emulsion is the high sensitive photographic film used for detection of three-dimensional trajectory of charged particles such as muons with the position resolution of sub-micron. A nuclear emulsion has following properties: high spatial resolution with thin detector structure, lightweight, flexibility of detector size and no requirement of electric power supply. These properties of a nuclear emulsion are suitable for a detector of muography. We are developing the nuclear emulsion at Nagoya University. In this paper, the current performances of the nuclear emulsion and the fully self-production techniques developed at Nagoya University are described.
\end{abstract}

\section{Introduction}

Muography (cosmic-ray muon radiography) is a visualization technique of interior density distribution of large-scale structures such as volcanoes [Tanaka et al. 2007a, 2007b, 2007c, 2009, 2010; Lesparre et al. 2012, Cârloganu et al. 2013, Carbone et al. 2014], the pyramid [Alvarez et al. 1970], nuclear reactors [Morishima et al. 2012, Fujii et al. 2013], and so on. Muons are produced from collisions of primary cosmic-rays, which consist mainly of proton, with the Earth's atmosphere as shown in Figure 1. Generated muons in this way, called cosmic-ray muons, come from all direction to the face of the Earth consistently and have broad energy distribution. High energetic muon has high penetration power that is able to penetrate through several $\mathrm{km}$ of rocks. The relation between muon energy and penetration length of the standard rock thickness [Groom et al. 2001] is shown in Figure 2. For example, a muon

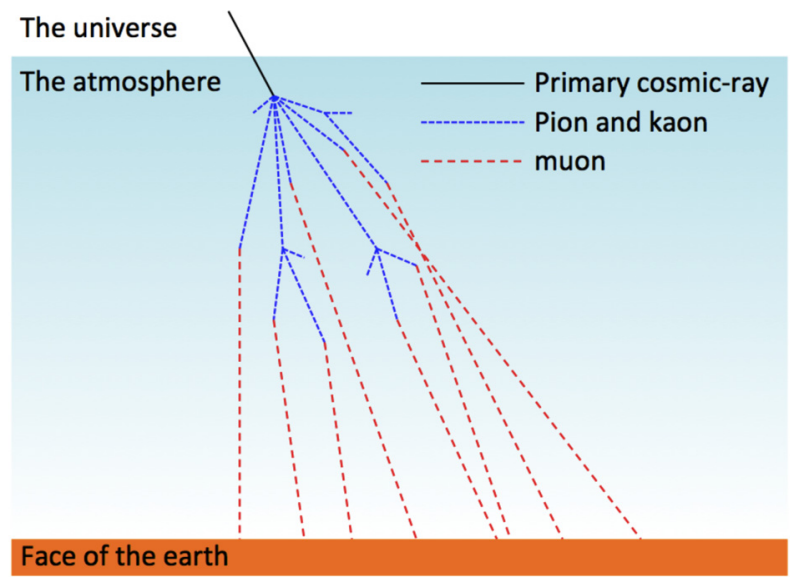

Figure 1. Production process of cosmic-ray muons. Electromagnetic components, nuclear components and neutrinos are removed in order to highlight the muon production process in this figure. Incoming muon rate on the face of the Earth is approximately 1 $\mathrm{muon} / \mathrm{min} / \mathrm{cm}^{2}$.

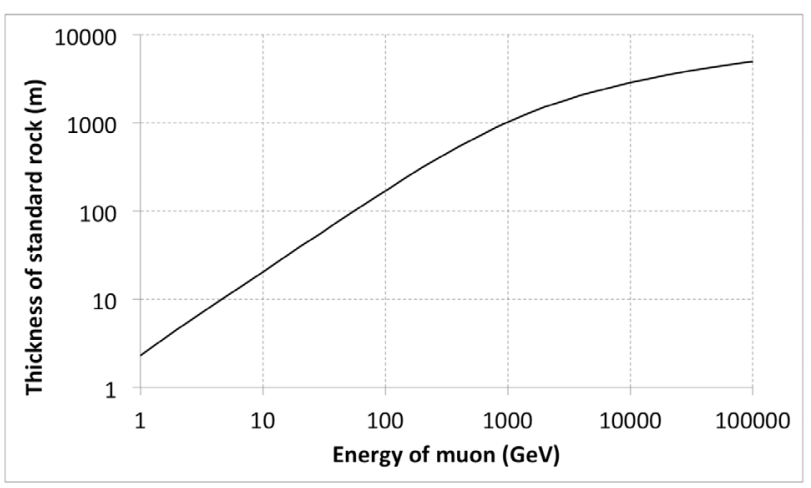

Figure 2. A relation between penetration thickness of standard rock and energy of muon [Groom et al. 2001].

with an energy of $1 \mathrm{TeV}$ is able to pass through $1 \mathrm{~km}$ of rocks. Thanks to the broad incoming muon energy dis- 


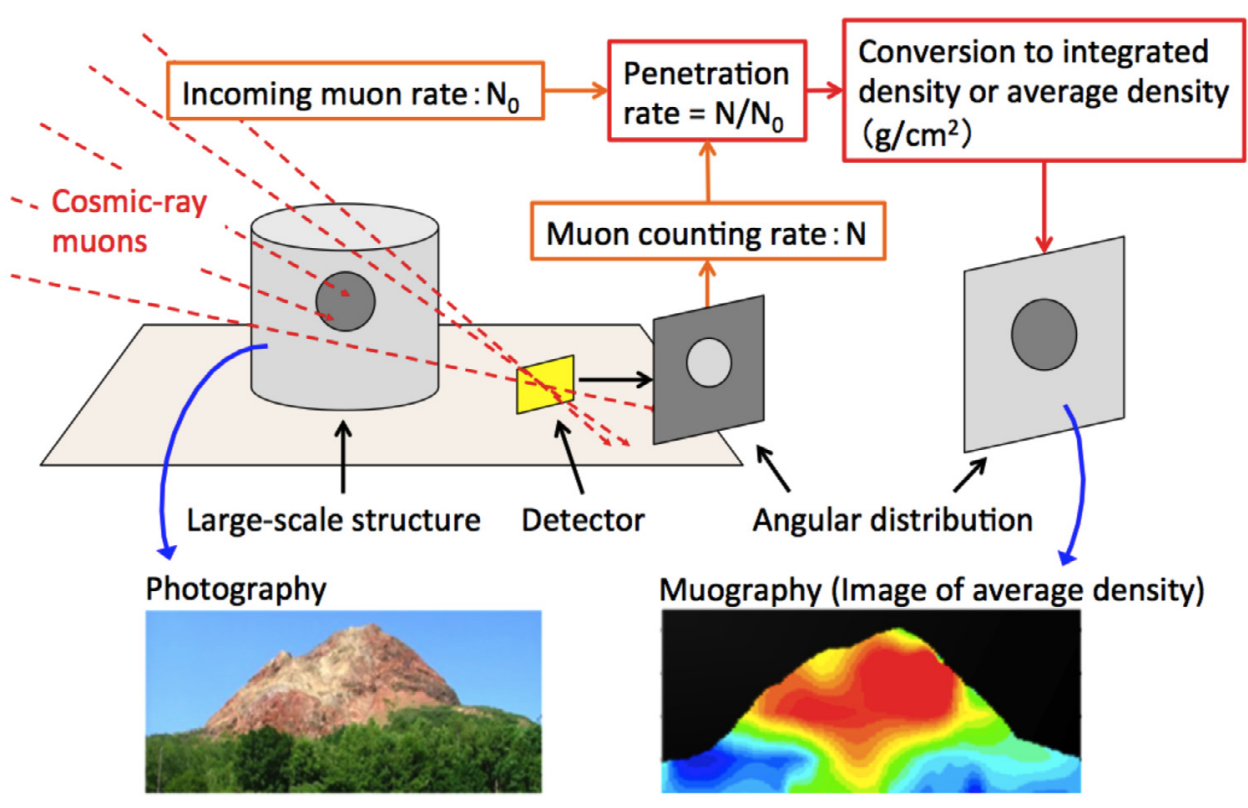

Figure 3. A schematic diagram of muography. A photography and a muography in the lower part of the figure are referenced from Tanaka et al. [2007a].

tribution, only muons with enough energy are able to penetrate material in the pathway and reach to the face of the Earth. The principle of muography, similar to Xray imaging technique, is the measurement of penetration rate of cosmic-ray muons pass through a large-scale structure and conversion to two-dimensional integrated density distribution as shown in Figure 3. In addition, if we know the three-dimensional shape of the structure, we can convert to average density distribution.

In comparison with other various non-destructive inspections, the muography has wide effective detection range and high spatial resolution thanks to properties of cosmic-ray muon. The request performance of muon detector for muography is detection of incoming angle. The required angular resolution to the detector is calculated by dividing required position resolution by the distance from observed object. Typically for enough spatial resolution, an angular resolution of a few mrad is needed. In addition, in order to take a high spatial resolution image, enough statistics of detected muons are necessary. Thus, a large area detector is required to collect a lot of muons because cosmic-ray muon rate on the face of the Earth is constant. From these kinds of reasons, requirements for muon detector are both high resolution to achieve enough spatial resolution and large area to achieve enough statistics.

\section{Nuclear emulsion}

A nuclear emulsion is a high sensitive photographic film sensitized to minimum ionizing particles (MIPs) such as cosmic-ray muons and is able to record three-dimensional particle trajectories with sub-micron spatial resolution as tracks when particle passes through in its sensitive emulsion layer as shown in Figure 4. These recorded tracks are detected by using an optical microscope. A nuclear emulsion is very old technology invented in the 1940s [Powell et al. 1959]. Nowadays, an automated high-speed nuclear emulsion read-out system called track selector (TS) based on an optical microscope with latest computing technologies were developed by our group [Niwa et al. 1974, Aoki et al. 1990, Nakano 1997, Morishima and Nakano 2010] from 1970s. The read-out system takes sixteen divided tomographic images along the direction of optical axis in the emulsion layer and searches a straight track. After track recognition, track data (position, angle, pulse height) is saved into the database. The read-out speed of emulsion layer was reached to approximately $100 \mathrm{~cm}^{2} / \mathrm{h}$ by super-ultra track selector (S-UTS) and will be reached to approximately $1 \mathrm{~m}^{2} / \mathrm{h}$ in near future by hyper track selector (HTS) under development.

In addition, we put the emulsion gel production machine into operation in 2010 at Nagoya University and are able to produce an emulsion gel independently. By using the production machine, we are able to improve and tune performances (sensitivity, long-term stability and so on) of a nuclear emulsion gel for purposes. In addition, we are developing the pouring technique and the mass production method. In this way, we are developing fully self-made emulsion technologies in laboratory level.

A nuclear emulsion has the following advantages for muography: High spatial resolution (position resolution is sub-micron, angular resolution is a few mrad) with very thin detector structure, lightweight, flexibility of detector size from $1 \mathrm{~cm}$ length to $1 \mathrm{~m}$ length and 


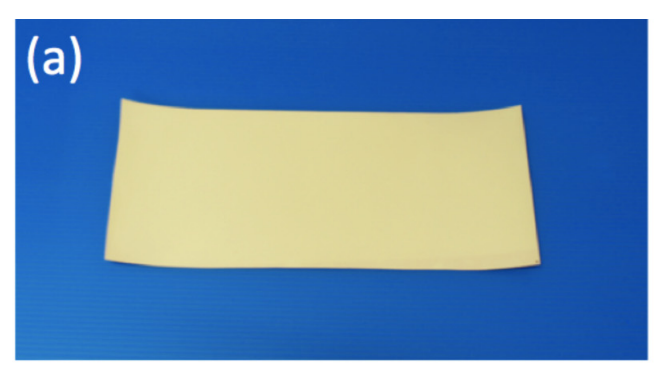

(b)

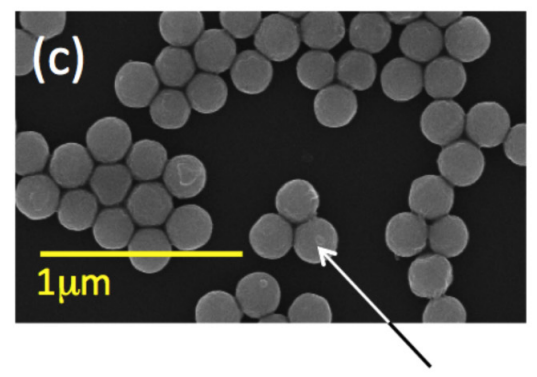

(d)

silver bromide crystals
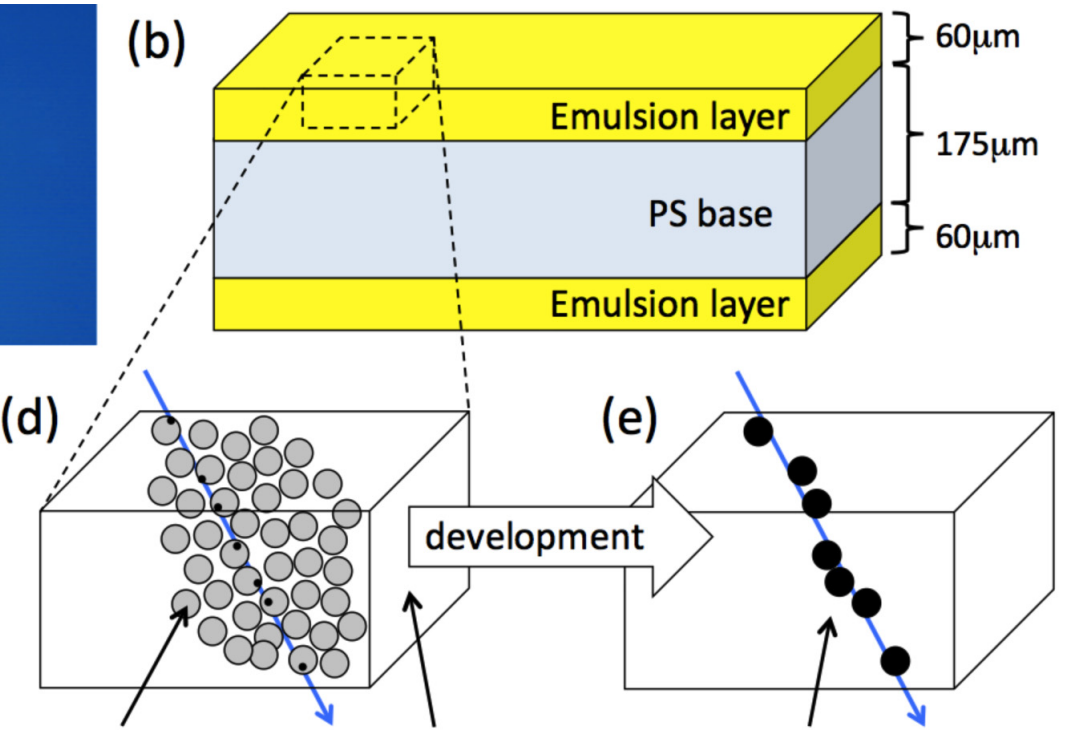

gelatin layer

3-dimensional track

Figure 4. (a) A picture of the nuclear emulsion; (b) An Illustration of the structure of the nuclear emulsion; (c) An electron microscope image of silver bromide crystals of the nuclear emulsion. The diameter of crystals is approximately $200 \mathrm{~nm}$; (d) and (e) The principle of detection of a charged particle in the nuclear emulsion. Black small dots on silver bromide crystals show latent images in (d).

no requirement of electric power supply. These properties are ideal for muography in various environments due to portability, scalability and flexibility.

\subsection{Structure of nuclear emulsion}

The picture of Figure $4 \mathrm{a}$ is the nuclear emulsion we produced and Figure $4 \mathrm{~b}$ shows the structure of the nuclear emulsion. An emulsion gel is poured on the $170 \mu \mathrm{m}$ transparent polystyrene (PS) plastic base, which acts as a supporting material. A huge amount of silver bromide (AgBr) crystals, which diameter is $200 \mathrm{~nm}$, are dispersed in $60 \mu \mathrm{m}$ gelatin layer coated on both sides of PS base. The picture of Figure $4 \mathrm{c}$ is an electron microscope image of $\mathrm{AgBr}$ crystals. A charged particle passes through the emulsion layer and some crystals in the line of the trajectory have a latent image as shown in Figure $4 \mathrm{~d}$. After chemical development process, these latent images become sub-micron silver particles (grains) as shown in Figure 4e. The grain size in an optical microscope image is approximately $1 \mu \mathrm{m}$ and the size is sufficiently smaller than the thickness of the emulsion layer. Thanks to the structure, a trajectory of a charged particle (track) can be recognized in three-dimensions with sub-micron accuracy. An angular range of data taking of tracks is depends on the recognition algorithm of the read-out system. The angular range of the TS is 45 degrees in an ordinary condition.

\subsection{Production of emulsion film}

We established nuclear emulsion production techniques as shown in Figure 5. We are able to produce an emulsion gel for R\&D and small amount mass produc- tion by using $0.5 \mathrm{~L}$ scale machine. In addition, we introduced $1.5 \mathrm{~L}$ scale machine for mass production in 2014 at Nagoya University. By using the new machine, we are able to produce a nuclear emulsion gel at the speed of approximately $3 \mathrm{~L} /$ day.

The pouring procedure constructed at Nagoya University is shown in Figure 5. We pour an emulsion gel inside a frame made of a vinyl tape and spread an emulsion gel with keeping the uniformity of the emulsion layer thickness by using the special glass coating tool. After an emulsion gel becomes solid state (setting), we move emulsion plates to a drying shelf. We process both side of an emulsion plate. The achieved uniformity of the thickness by this method is approximately $10 \mu \mathrm{m}$ in the area of $10 \mathrm{~cm} \times 10 \mathrm{~cm}$. After that, we perform cutting, humidity conditioning and packing with aluminum-laminated film. An aluminum-laminated film acts as shielding from water, shielding from light and holding water in the nuclear emulsion. By these methods, we can produce nuclear emulsions at the speed of $4 \mathrm{~m}^{2} /$ week. The production speed is limited by emulsion setting time and spaces of the shelf. For muography, $12.5 \mathrm{~cm} \times 30 \mathrm{~cm}$ size nuclear emulsions, which is shown in Figure 4a, are produced and used.

\subsection{Performance}

The track recognition efficiency of muons by the read-out system strongly depends on the density of grains per length along the line of a track. The number of grains per 100 microns is called grain density (GD). In order to increase GD, we tuned the volume occupancy of AgBr crystals of 55\% in the nuclear emulsion 


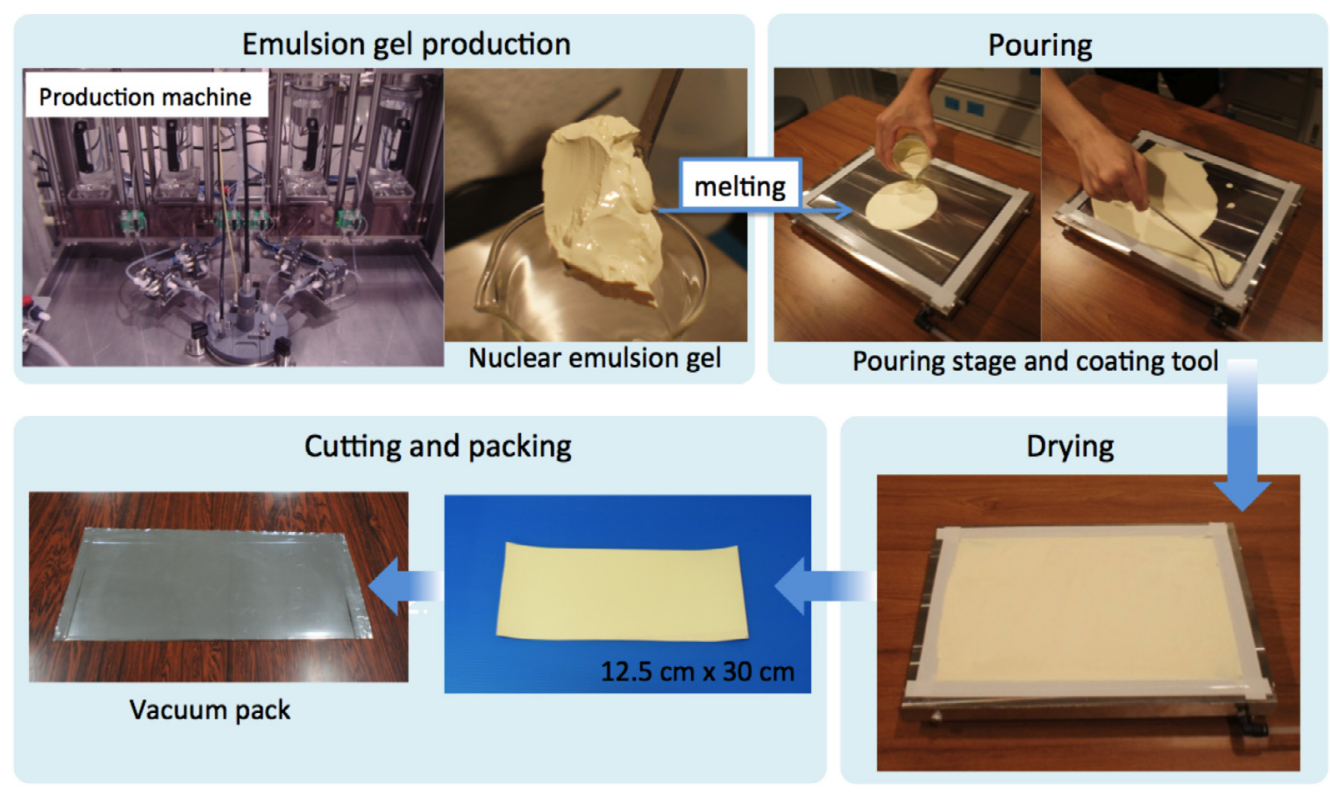

Figure 5. An overview of a process of self-made production of the nuclear emulsions.

layer. In addition, we tuned amount of a few additive chemicals (surfactant, plasticizer and hardener) for achievement of sufficient flexibility in order to prevent an emulsion layer from cracking. The optical microscope image of one of MIP track in the nuclear emulsion is shown in Figure 6. The GD of the nuclear emulsion is $51 \pm 2$ by counting an image taken by an image sensor by eye. The nuclear emulsion evaluated was developed with a developer for OPERA experiment (PD-T) produced by Fuji Photo Film Co., Ltd. for 25 minutes at 20 degrees Celsius. The GD is higher than that of OPERA film [Nakamura et al. 2006], which is produced for the OPERA experiment and used for muography in the past [Tanaka et al. 2007a, 2007b, 2007c; Morishima et al. 2012].

The pulse height $(\mathrm{PH})$ distribution of cosmic-ray tracks of its angle $|\tan \theta|<0.1$ recorded in the emulsion layer taken by the S-UTS [Morishima and Nakano 2010], which is one of the TS, is shown in Figure 7a. The angle $\theta$ is defined by an angle between a track angle and an optical axis of the read-out system. The number of tomographic layers is 16 and the $\mathrm{PH}$ is the number of layers, which includes grains of a track. This $\mathrm{PH}$ distribution enables us to achieve a track recognition efficiency of more than $97 \%$ in a normal radiation background.

The angular resolution of cosmic ray tracks of its angle $|\tan \theta|<0.1$ recorded in the emulsion layer taken

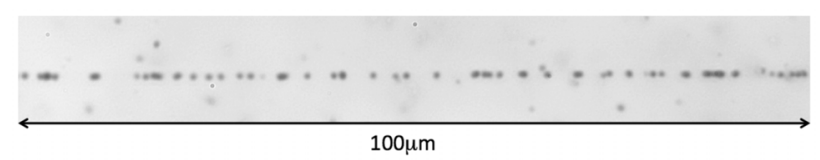

Figure 6. A microscope image of a track of minimum ionizing particle in the nuclear emulsion. Black dots are silver gains. A chain of silver grains is a trajectory of charged particle.

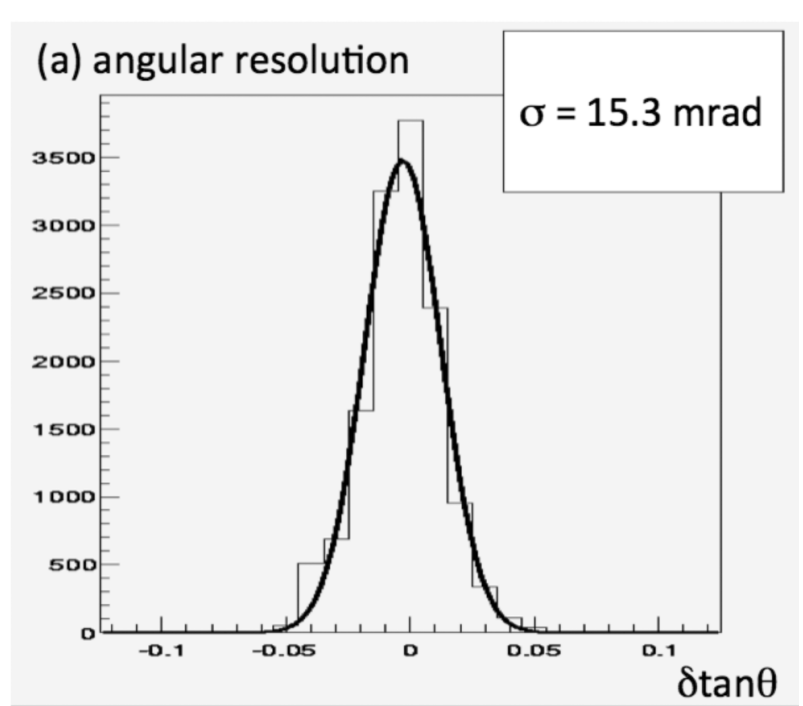

(b) $\mathrm{PH}$ distribution

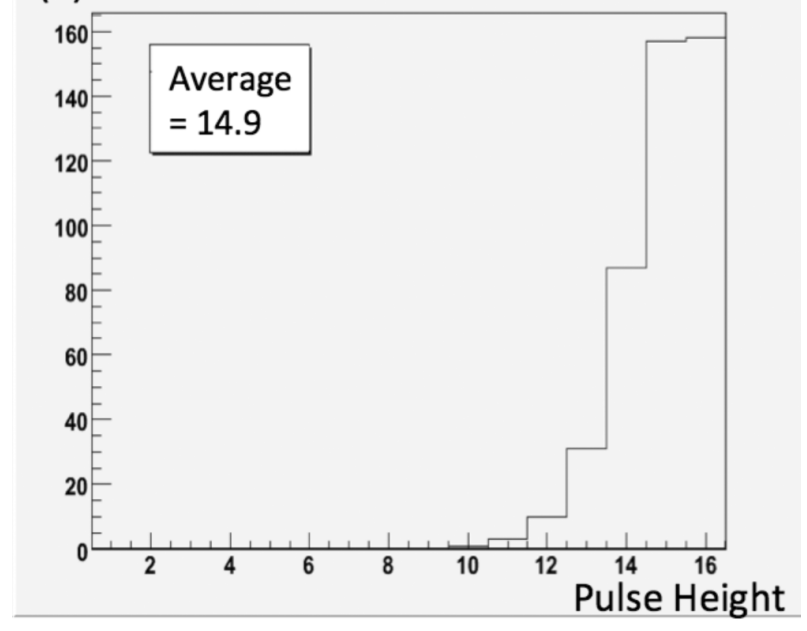

Figure 7. (a) A histogram of angular resolution of tracks recorded in the emulsion layer; (b) A histogram of the pulse height ( $\mathrm{PH})$ distribution of tracks recorded in the emulsion layer. 
by the S-UTS is shown in Figure $7 \mathrm{~b}$. This resolution is equal to the position resolution of $0.45 \mu \mathrm{m}$ on the surface of the PS base. This position resolution gives us to achieve the angular resolution of a few mrad by using the track positions on both surfaces of the PS base.

The long time stability of the nuclear emulsion is important for muography. Especially, the temperature dependency of the latent image fading is very important because the latent image fading is accelerated by high temperature. The fading property of the nuclear emulsion in the combination of temperature $(25$ and 35 degrees Celsius) and relative humidity $(20 \%$ and $50 \%$ ) is shown in Figure 8. In this evaluation, a time variation rate of fading effect, which is measured GD at each sampling date divided by initial GD (relative sensitivity), is presented. The result shows that high temperature and high relative humidity of the nuclear emulsion accelerate decrease of GD. Thus, we need to decide an observation period according to the surrounding temperature with controlling relative humidity of the nuclear emulsion in an aluminum laminated film package in the observation place.

\subsection{Observation}

The structure of nuclear emulsion detector is optimized for various observation surroundings. The nuclear emulsion is attached on a flat plate, for example an aluminum plate, as shown in Figure 9a. In this case, the nuclear emulsion is fixed in the vertical direction to the ground in order to measure incoming muons in a low elevation angle, for example to measure volcanoes. In order to reflect sunlight and suppress a rise in temperature, the nuclear emulsion is put in the thermal insulation covered with light reflective material on case-by-case basis as shown in Figure 9b. The nuclear emulsion is fixed in the parallel direction to

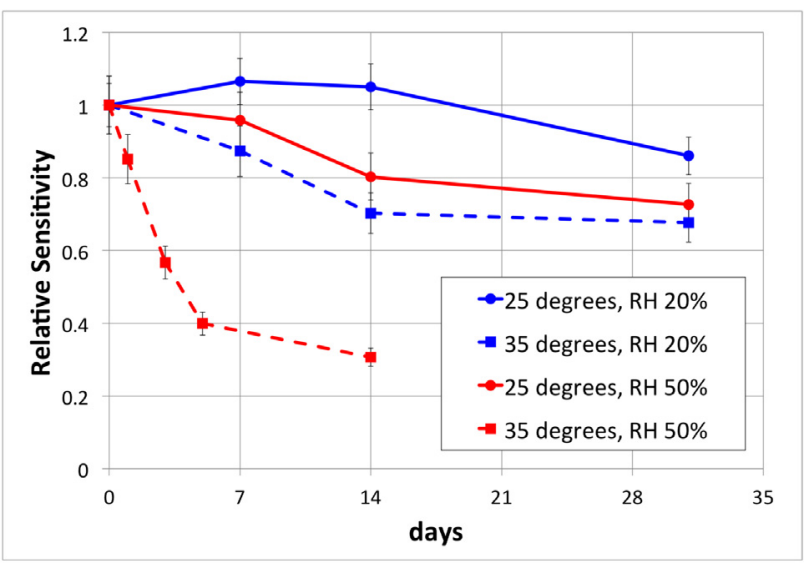

Figure 8. The fading property of the nuclear emulsion. A relative humidity is written as $\mathrm{RH}$ in this figure. In the case of combination between 35 degrees and RH 50\%, measurement dates are 1, 3, 5, 14 days. In the other case, measurement dates are 7, 14, 31 days.
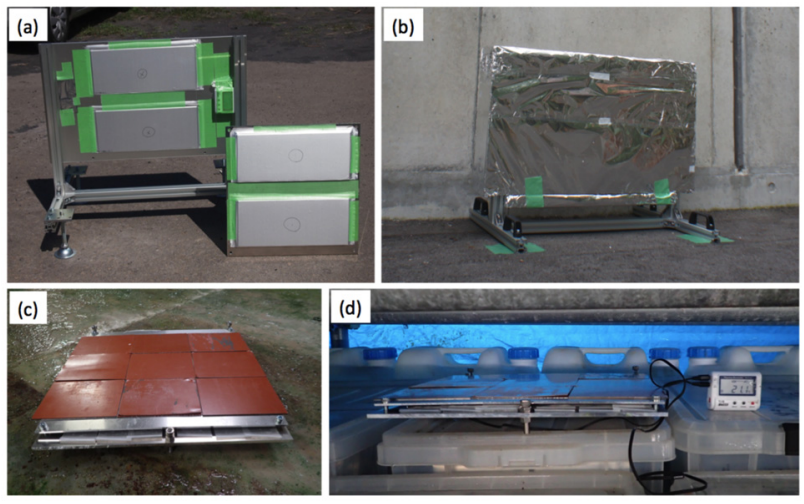

Figure 9. Pictures of nuclear emulsion detectors. (a) A picture of a structure of the nuclear emulsion detector fixed in the vertical direction to the ground; (b) A picture of the detector, which is same type as (a), covered by the thermal insulation with light reflective material; (c) A a picture of the nuclear emulsion detector fixed in the parallel direction to the ground; (d) is a picture of the nuclear emulsion detector of a picture (c) placed in a narrow space.

the ground in order to measure incoming muons from zenith direction, for example to search for unknown empty space in the upper direction as shown in Figure $9 \mathrm{c}$. Nuclear emulsions are fixed inside the supporting aluminum material with lead plates to shield from gamma ray emitted from surrounding bedrock and the thickness of the structure is kept in approximately $5 \mathrm{~cm}$ in order to install in a narrow space as shown in Figure 9d. The main application of nuclear emulsions produced at Nagoya University is the measurement of Fukushima Daiichi Nuclear Power Plant, which was conducted from 2014 to 2015, until now. This is the first experiment using the high sensitive nuclear emulsion produced at Nagoya University. In addition, we start to apply nuclear emulsions in various fields such as geoscience, archaeology, civil engineering and so on.

\section{Conclusion}

Nuclear emulsion has ideal properties for a detector of muography. We developed the nuclear emulsion production technology and improved the muon detection efficiency. The production speed is approximately $4 \mathrm{~m}^{2} /$ week by our hands. The sensitivity (GD) of the nuclear emulsion is higher than that of OPERA film and enables us to achieve the efficiency of more than $97 \%$ by the S-UTS. The angular resolution of a track is $15 \mathrm{mrad}$ in the emulsion layer. The accuracy is almost the same as that of the OPERA film. These performances are sufficiently for a detector of muography. The nuclear emulsion we developed is already applied to muography of Fukushima Daiichi Nuclear Power Plant and are beginning to be widely applied to other fields such as geoscience, archaeology, civil engineering and so on. 


\section{References}

Alvarez, L.W., J.A. Anderson, F. El Bedwei, J. Burkhard, A. Fakhry, A. Girgis, A. Goneid, F. Hassan, D. Iverson, G. Lynch, Z. Miligy, A.H. Moussa, MohammedSharkawi and L. Yazolino (1970). Search for Hidden Chambers in the Pyramids, Science, 167, 832-839.

Aoki, S., K. Hoshino, M. Nakamura, K. Niu, K. Niwa and N. Torii (1990). Fully Automated Emulsion Analysis System, Nucl. Instum. Meth. B, 51, 466-472.

Carbone, D., D. Gibert, J. Marteau, M. Diament, L. Zuccarello and E. Galichet (2013). An experiment of muon radiography at Mt Etna (Italy), Geophys. J. Int., 196 (2), 633-643; doi:10.1093 / gji/ggt403.

Cârloganu, C., et al. (2013). Towards a muon radiography of the Puy de Dôme, Geosci. Instrum. Meth., 2, 55-60.

Fujii, H., K. Hara, S. Hashimoto, F. Ito, H. Kakuno, S.H. Kim, M. Kochiyama, K. Nagamine, A. Suzuki, Y. Takada, Y. Takahashi, F. Takasaki and S. Yamashita (2013). Performance of a remotely located muonradiography system to identify the inner structure of a nuclear plant, Prog. Theor. Exp. Phys., 073C01.

Groom, D.E., N.V. Mokhov and S.I. Striganov (2001). Muon stopping muon stopping power and range tables $10 \mathrm{MeV}-100 \mathrm{TeV}$, Atom. Data Nucl. Data, 78, 183-356.

Lesparre, N., D. Gibert, J. Marteau, J.C. Komorowski, F. Nicollin and O. Coutant (2012). Density muon radiography of La Soufrière of Guadeloupe volcano: comparison with geological, electrical resistivity and gravity data, Geophys. J. Int., 190, 1008-1019.

Morishima, K., and T. Nakano (2010). Development of a new automatic nuclear emulsion scanning system, S-UTS, with continuous 3D tomographic image read-out, J. Instrum., 5, P04011.

Morishima, K., et al. (2012). First demonstration of cosmic ray muon radiography of reactor cores with nuclear emulsions based on an automated high-speed scanning technology, In: Proceedings of the 26th Workshop on Radiation Detectors and Their Uses in KEK, 27-36.

Nakamura, T., et al. (2006). The OPERA film: New nuclear emulsion for large-scale, high-precision experiments, Nucl. Instum. Meth. A, 556, 80-86.

Nakano, T. (1997). Automatic analysis of nuclear emulsion, Ph.D. Thesis, Nagoya University, Japan.

Niwa, K., K. Hoshino and K. Niu (1974). Auto scanning and measuring system for the emulsion chamber, In: Proceedings of the International Cosmic Ray Symposium on High Energy Phenomena, Cosmic Ray Laboratory, University of Tokyo, Japan, 149.

Powell, C.F., P.H. Fowler and D.H. Perkinset (1959). The study of elementary particles by the photographic method; an account of the principal techniques and discoveries, illustrated by an atlas of photomicrographs, London, New York, Pergamon Press.

Tanaka, H.K.M., T. Nakano, S. Takahashi, J. Yoshida, H. Ohshima, T. Maekawa, H. Watanabe and K. Niwa (2007a). Imaging the conduit size of the dome with cosmic ray muons: The structure beneath Showa Shinzan Lava Dome, Japan, Geophys. Res. Lett., 34, L22311.

Tanaka, H.K.M., T. Nakano, S. Takahashi, J. Yoshida, M. Takeo, J. Oikawa, T. Ohminato, Y. Aoki, E. Koyama, H. Tsuji and K. Niwa (2007b). High resolution imaging in the inhomogeneous crust with cosmic ray muon radiography: The density structure below the volcanic crater floor of Mt. Asama, Japan, Earth Planet. Sc. Lett., 263, 104-113.

Tanaka, H.K.M., T. Nakano, S. Takahashi, J. Yoshida and K. Niwa (2007c). Development of an emulsion imaging system for cosmic-ray muon radiography to explore the internal structure of a volcano, $\mathrm{Mt}$. Asama, Nucl. Instrum. Meth. A, 575, 489-497.

Tanaka, H.K.M., T. Uchida, M. Tanaka and H. Shinohara (2009). Cosmic-ray muon imaging of magma in a conduit: Degassing process of Satsuma-Iwojima Volcano, Japan, Geophys. Res. Lett., 36, L01304, 1-5.

Tanaka, H.K.M., H. Taira, T. Uchida, M. Tanaka, M. Takeo, T. Ohminato, Y. Aoki, R. Nishitama, D. Shoji and H. Tsuiji (2010). Three-dimensional computational axial tomography scan of a volcano with cosmic ray muon radiography, J. Geophys. Res., 115, B12332.

\footnotetext{
${ }^{\star}$ Corresponding author: Kunihiro Morishima, Institute for Advanced Research (IAR), Nagoya University, Nagoya, Japan; email: morishima@flab.phys.nagoya-u.ac.jp.

C 2017 by the Istituto Nazionale di Geofisica e Vulcanologia. All rights reserved.
} 